\title{
Treatment patterns and outcomes for patients with advanced melanoma in US oncology clinical practices
}

\author{
Eric D Whitman ${ }^{1}$, Frank Xiaoqing Liu*,2, Xiting $\mathrm{CaO}^{2}$, Scott J Diede ${ }^{2}$, Amin Haiderali $^{2}$ \& \\ Amy $P$ Abernethy ${ }^{3}$ \\ ${ }^{1}$ Atlantic Melanoma Center, Atlantic Health System Cancer Care, 100 Madison Ave., Morristown, NJ 07960, USA \\ ${ }^{2}$ Merck \& Co., Inc., 2000 Galloping Hill Rd, Kenilworth, NJ 07033, USA \\ ${ }^{3}$ Flatiron Health, Inc., 233 Spring St, New York, NY 10013, USA \\ *Author for correspondence: Tel.: +1 267305 1265; xiaoqing.liu@merck.com
}

\begin{abstract}
Aim: To describe recent evolution in treatment patterns and outcomes for advanced melanoma (AMel). Methods: This retrospective observational study analyzed de-identified electronic health record data from the Flatiron Health database for 1140 adult patients who initiated first-line therapy for AMel from 1 January 2014 to 30 June 2016 with follow-up through 28 February 2017. Results: The most common firstline regimens were ipilimumab-based therapies (34\%), anti-PD-1 monotherapy (26\%) and BRAF/MEK inhibitor(s) (20\%). First-line ipilimumab-based and BRAF inhibitor regimens decreased after the third quarter of 2014 (3Q2014), and by 2Q2016, 55 and $91 \%$ of BRAF-mutant and BRAF wild-type cohorts, respectively, received a first-line anti-PD-1 regimen. Median overall survival from first-line initiation for all patients was 18.8 months (95\% Cl: 16.3-23.3). Conclusion: Results illustrate changing paradigms of therapy and real-world patient outcomes for AMel.
\end{abstract}

First draft submitted: 14 August 2018; Accepted for publication: 10 September 2018; Published online: 25 September 2018

Keywords: advanced melanoma • antineoplastic therapy • community oncology practice • immuno-oncology • observational study • overall survival • treatment pattern

The incidence rate of melanoma in the US has doubled in the past 40 years, a more rapid increase than for any other type of cancer [1,2], and similar trends have been observed in Europe and Australia [2,3]. Cutaneous melanoma represents $5.3 \%$ of all new cancer cases and is projected to be the fifth leading cause of cancer in the US in $2018[4,5]$. Historically, the median survival time for patients with advanced, unresectable (stage IV) melanoma was only 6-9 months, although this estimate is now becoming outdated with the introduction of newer therapies [2].

For many years, the primary systemic treatments for advanced melanoma (AMel) were chemotherapy - such as dacarbazine - and immunotherapy - such as IL-2. These therapies had low overall response rates ranging from 7 to $16 \%$, often accompanied by associated side effects, and provided limited to no benefits on survival [6-9]. Indeed, no progress in improving survival rates was recorded during the last three decades of the 20th century [10]. Nonetheless, the potential of immunotherapy to treat melanoma has long been recognized, as high dose IL-2 can induce long-term remission, potentially cures, in a small number of patients with metastatic disease [11,12].

The promise of new immunotherapy agents and discoveries of isolated and treatable tumor-specific genetic mutations have now dramatically altered the landscape for systemic therapy of AMel. The start of a new era was heralded by the US FDA's approval of ipilimumab, an anti-CTLA-4 in March 2011, followed by approvals of targeted BRAF/MEK inhibitors, including vemurafenib, dabrafenib, trametinib and cobimetinib, and then two monoclonal antibodies directed against the PD-1 receptor, pembrolizumab and nivolumab in September 2014 and December 2014, respectively. These agents have all demonstrated significant improvements in progression-free and/or overall survival (OS) for patients with AMel in randomized controlled clinical trials [13-21].

Targeted therapy with BRAF/MEK inhibitors is indicated for patients with a BRAF V600 mutation, who comprise approximately $45 \%$ of patients with AMel $[2,22,23]$. The most recent data strongly suggest that combined therapy with BRAF and MEK inhibitors is more effective than monotherapy with either type of agent [24-26].

Future $\because$ Medicine 
Treatments with these agents are characterized by rapid tumor shrinkage that is generally time limited, as the tumors often develop resistance [24,25]. Treatments with the so-called 'checkpoint inhibitors', such as ipilimumab, pembrolizumab and nivolumab, are associated with lower (but still impressive) response rates and less predictable kinetics of response but appear to have the potential for long-term clinical remissions and benefit $[14,20,21,27]$. Despite a fairly low-response rate and potentially severe toxicity with ipilimumab monotherapy, some patients can derive long-term clinical benefit and survival [28,29]. Anti-PD-1 therapy has demonstrated higher response rates with lower toxicity than ipilimumab although many patients do not respond [20,30-32]. Most recently, the combination of ipilimumab and nivolumab was approved by the FDA in 2016; however, its potential dramatic efficacy is offset by a heightened risk of toxicity [33,34].

The treatment of melanoma, more than that for any other cancer, has evolved so quickly over the past few years that it has been difficult for regulatory agencies, insurance payers and guideline committees to keep pace [35]. Yet the biggest challenge faced by physicians and patients is to select a treatment strategy from the lengthening list of effective therapies for what was previously a dismal prognosis. Additionally, patients seen in clinical practice may differ from those treated in randomized controlled clinical trials [36], and real-world evidence to complement trial findings can be useful to understand the outcomes of therapy in clinical practice [37]. The aims of this retrospective observational study were to understand the evolution of treatment patterns, time to next treatment line and associated OS for patients with AMel in US oncology clinical practices following the approval of these new therapies.

\section{Methods}

\section{Data source}

Our study population was identified from the Flatiron Health cloud-based longitudinal database derived from electronic health record (EHR) data from US cancer clinics [38]. At the time of the study, the Flatiron database contained EHR data available for analysis from over 265 cancer clinics across approximately 800 sites of care, including the active records of more than 1.7 million patients with cancer in the four US census regions. Refreshed monthly, the patient-level data are abstracted from EHRs and include structured data, such as laboratory values, limited biomarker information and prescribed drugs, in addition to unstructured data taken from physician's notes in the medical record and other unstructured documents (e.g., detailed biomarker, radiology and pathology reports).

We included patients from the Flatiron AMel cohort (March 2017 analytic dataset, with mortality v1.0) [38], which includes patients with cutaneous melanoma at pathologic stage III or IV, either at initial diagnosis or at local or distant recurrence, and with at least two clinic encounters evident in the database occurring on or after 1 January 2011. To construct the cohort, a random, representative sample of patients is selected using a computer-based algorithm, and manual review of structured and unstructured data is conducted to ensure that those patients have the diagnosis of AMel. The diagnosis of melanoma is confirmed by review of pathology reports (International Classification of Diseases, Ninth Revision, Clinical Modification codes [ICD-9-CM] 172.0 through 172.9) [39], and melanoma is staged according to the treating physician's charted notes plus radiology, pathology and surgical reports. Patients with noncutaneous melanoma (e.g., ocular, subungual, mucosal, palmar, plantar) are excluded.

Institutional review board approval of the study protocol was obtained through Flatiron procedure and approved by Copernicus Group institutional review board before study conduct and included a waiver of informed consent. Data provided to third parties were de-identified and provisions were in place to prevent re-identification in order to protect patients' confidentiality.

\section{Patients}

We included adult patients ( $\geq 18$ years) with a confirmed diagnosis of AMel who initiated first-line therapy for AMel from 1 January 2014, through 30 June 2016. These patients were followed until their last available medical record or to 28 February 2017, thereby providing minimum 8 months' possible follow-up for each patient from the start of first-line therapy. Evidence in the structured data of enrollment in a clinical trial at any time was cause for exclusion.

\section{Data variables}

Demographic variables available from the Flatiron database included sex, birth year, race and height and weight; age and BMI were derived from these data. Disease-related variables included melanoma stage at diagnosis, dates of initial melanoma and AMel diagnoses, Eastern Cooperative Oncology Group performance status (ECOG PS) 
and presence of brain metastases (ICD-9-CM diagnosis code 198.3) and date. The Charlson Comorbidity Index score was derived from ICD-9 diagnostic data [40]; however, the Charlson Comorbidity Index was scored without consideration of AMel diagnosis since that was an entry criterion. The timing and results of $B R A F$ mutation testing were captured by reviewing the relevant biomarker reports. Mortality data were captured through a combination of structured EHR data, unstructured EHR data (such as clinician notes and condolence letters), and external death data sources, including the US Social Security death index [41,42] and a commercial death dataset.

Treatment-related variables available in the database included drug names, route, dose and units, and the lines of therapy were determined by applying predefined algorithms. The first line of therapy for AMel was defined as the first systemic treatment regimen beginning either after or $<14$ days before the AMel diagnosis, and the index date was defined as the date of initiation of first-line therapy. Regimen components that began within 28 days of the first episode were considered to be part of a single line of therapy. A treatment line was advanced to the next line when a patient received new combinations of drugs or there was a gap in drug orders or administrations of $>120$ days. The line of therapy was not advanced if chemotherapy combinations were followed by a similar regimen in which one or more of the component drugs were suppressed for a period of time and then the drug(s) were subsequently reintroduced. A review of records indicated that treatments could change within the first month of initiation upon receipt of biomarker results. Therefore, if patients were switched to BRAF-targeted therapy during the first 28 days of a chemotherapy regimen (monotherapy or combination), the line of therapy was called BRAF therapy and the line number was not advanced. Not all Flatiron records contained days of supply data for oral drugs; when the information was missing, we assumed that the last prescription had 30 days' supply.

\section{Statistical analysis}

Descriptive analyses were conducted to describe the patient population and treatment patterns, overall and according to BRAF mutation status at the index date. We determined frequencies and percentages for categorical variables and mean, standard deviation, median and range for variables measured on the continuous or interval scale. Patient characteristics and treatment patterns were described for all patients as well as according to BRAF status at the index date. The follow-up time was reported by summary statistics, and the median time to next treatment was reported using the Kaplan-Meier method. If a patient did not die nor initiate a next line of therapy, then this patient was considered still on the same line of therapy and was censored in the Kaplan-Meier estimate for time to next treatment line on the last visit date. OS was described using the Kaplan-Meier method, reporting median OS and associated $95 \%$ CI. Kaplan-Meier estimates of the percentage of patients on any therapy at 1 and 2 years were also reported.

Statistical analyses were performed using SAS 9.4 software (SAS Institute, Inc., NC, USA).

\section{Results}

\section{Patients}

Of the 4413 patients with a diagnosis of AMel from 2011 onward who were included in the Flatiron AMel cohort, 2027 (46\%) initiated first-line systemic therapy and 1181 (27\%) initiated the first line of systemic therapy during the study period from 1 January 2014 through 30 June 2016; all were $\geq 18$ years of age. After excluding 41/1181 patients (3\%) enrolled in clinical trials, the protocol-defined study cohort included 1140 eligible patients (97\%) with AMel (Figure 1); of the 1140 patients, 78 (7\%) were seen at academic centers and 1062 (93\%) at community oncology clinics.

The median age at the index date was 68 years (range: $20-84$ years): $68 \%$ of patients were male and $91 \%$ were white (excluding those with missing data; Table 1). The median ages were 63 and 71 years in BRAF-mutant and $B R A F$ wild-type cohorts, respectively, and the sex distribution was 67 and $70 \%$ male, respectively.

Most patients (1037; 91\%) were tested for $B R A F$ mutation, and most patients (1014; 89\%) had known $B R A F$ mutation status, including 454 (45\%) with $B R A F$-mutant melanoma and $560(55 \%)$ with $B R A F$ wild-type melanoma (Table 1). The 454 BRAF-mutant melanomas included 261 (57\%), 68 (15\%) and 24 (5\%) with BRAF V600E, BRAF V600K and other BRAF mutation types, respectively; information on BRAF mutation type was unknown or missing for $101(22 \%)$ tumors. The test types included PCR (43\%), sequencing $(35 \%)$ and other (missing or unknown, 22\%).

Most patients who developed AMel received a first diagnosis of melanoma at stage III or later, including 327 patients (37\%) overall with diagnosis at stage IV (Table 1). Of those with available data, 91 patients (17\%) had ECOG PS of 2 or greater. An elevated LDH level at the index date was recorded for 29 and $22 \%$ of patients in 


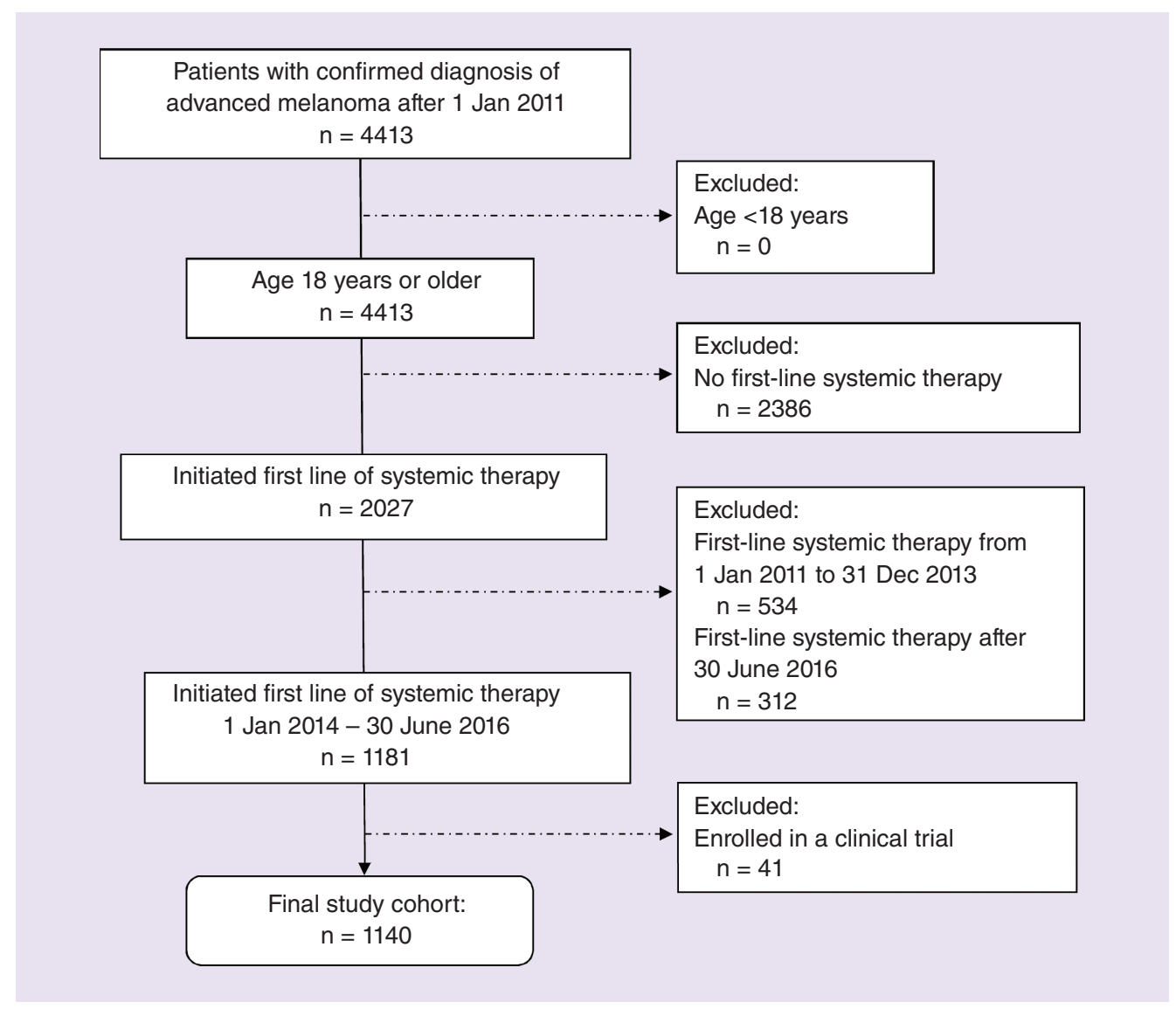

Figure 1. Selection of eligible patients in the database.

$B R A F$-mutant and $B R A F$ wild-type cohorts, respectively, and history of brain metastases in 12 and $7 \%$, respectively (Table 1).

\section{Treatment patterns}

The median study follow-up was 9.9 months (range: 0.03-42.6 months) for all patients and 10.4 months in both $B R A F$-mutant and BRAF wild-type cohorts (Table 2). During follow-up, a total of 734 patients (64\%) received only one line of therapy, $290(25 \%)$ received two lines and $116(10 \%)$ received three or more lines, as summarized by $B R A F$ mutation status in Table 2 .

The most commonly prescribed first-line regimens during the study period were ipilimumab as monotherapy or in combination with other than an anti-PD-1 agent (ipilimumab-based, 34\%), anti-PD-1 monotherapy (26\%) and regimens including a BRAF inhibitor (20\%). The most commonly prescribed second-line regimens during the study were anti-PD-1 monotherapy (48\%) and regimens including a BRAF inhibitor (20\%; Table 3).

For all patients in the study (Figure 2A), the most common first-line regimens shifted during the study period from those including ipilimumab or BRAF inhibitors (49 and 28\%, respectively, in the first quarter of 2014) to anti-PD-1 monotherapy or anti-PD-1 combination therapy (47 and 26\% in the second quarter of 2016; percentages are rounded and may not be additive). The first-line treatment selected for patients with $B R A F$-mutated tumors shifted over the study period: the most common first-line regimen at the start of the study included a BRAF inhibitor (64\%), and by the end of the index period, BRAF inhibitors (33\%), anti-PD-1 monotherapy (30\%) and anti-PD-1 combination therapy (25\%) were utilized in similar proportions (Figure 2B). For BRAF wild-type melanoma (Figure 2C), an ipilimumab-based regimen was the most common first-line regimen at the start of the study, and by the end of the study, anti-PD-1 monotherapy (62\%) and anti-PD-1 combination therapy (29\%) were the most common regimens. 
Table 1. Patient demographic and clinical characteristics at the index date (initiation of first-line therapy).

\begin{tabular}{|c|c|c|c|c|}
\hline Variables & BRAF mutant $(n=454)$ & BRAF wild-type $(\mathrm{n}=560)$ & BRAF status unknown $(\mathrm{n}=126)$ & Total $(n=1140)$ \\
\hline Male sex, n (\%) & $303(66.7)$ & $393(70.2)$ & $80(63.5)$ & $776(68.1)$ \\
\hline Median (IQR) age, years: & $63(53-73)$ & $71(61-80)$ & $68(60-79)$ & $68(58-77)$ \\
\hline - Range & $25-84$ & $20-84$ & $24-84$ & $20-84$ \\
\hline$-<55$ years, $\mathrm{n}(\%)$ & $125(27.5)$ & $69(12.3)$ & $18(14.3)$ & $212(18.6)$ \\
\hline$-55-64$ years, $n(\%)$ & $115(25.3)$ & $111(19.8)$ & $33(26.2)$ & $259(22.7)$ \\
\hline$-65-74$ years, $\mathrm{n}(\%)$ & $113(24.9)$ & $151(27.0)$ & $26(20.6)$ & $290(25.4)$ \\
\hline$-\geq 75$ years, $n(\%)$ & $101(22.2)$ & $229(40.9)$ & $49(38.9)$ & 379 (33.2) \\
\hline Race data available, n (\%): & $392(86.3)$ & 481 (85.9) & $104(82.5)$ & $977(85.7)$ \\
\hline - White, $\mathrm{n}(\%)^{\dagger}$ & $362(92.3)$ & $441(91.7)$ & $86(82.7)$ & $889(91.0)$ \\
\hline - Other, $\mathrm{n}(\%)^{\dagger}$ & $30(7.7)$ & $40(8.3)$ & $18(17.3)$ & $88(9.0)$ \\
\hline Mean (SD) weight, ${ }^{\ddagger} \mathrm{kg}$ & $85.0(20.7)$ & $83.3(19.0)$ & $81.6(18.2)$ & $83.8(19.6)$ \\
\hline US CB region, data available, $n(\%)$ : & $427(94.1)$ & $516(92.1)$ & $112(88.9)$ & $1055(92.5)$ \\
\hline - Northeast, $\mathrm{n}(\%)^{\dagger}$ & $66(15.5)$ & $104(20.2)$ & $16(14.3)$ & $186(17.6)$ \\
\hline - Midwest, n $(\%)^{\dagger}$ & $120(28.1)$ & $114(22.1)$ & $28(25.0)$ & $262(24.8)$ \\
\hline - South, n (\%) ${ }^{\dagger}$ & $159(37.2)$ & $209(40.5)$ & $50(44.6)$ & $418(39.6)$ \\
\hline - West, $\mathrm{n}(\%)^{\dagger}$ & $82(19.2)$ & $89(17.2)$ & $18(16.1)$ & $189(17.9)$ \\
\hline Adjusted CCl score, mean (SD): & $2.9(3.2)$ & $2.8(3.1)$ & $3.1(3.3)$ & $2.9(3.2)$ \\
\hline - Median (IQR) & $1(0-6)$ & $1(0-6)$ & $2(0-6)$ & $1(0-6)$ \\
\hline - Range & $0-13$ & $0-12$ & $0-11$ & $0-13$ \\
\hline ECOG PS data available, $n(\%)$ : & $211(46.5)$ & $258(46.1)$ & $74(58.7)$ & $543(47.6)$ \\
\hline - ECOG 0-1, n (\%) ${ }^{\dagger}$ & $177(83.9)$ & $214(82.9)$ & $61(82.4)$ & $452(83.2)$ \\
\hline$-\mathrm{ECOG} \geq 2, \mathrm{n}(\%)^{\dagger}$ & $34(16.1)$ & $44(17.1)$ & $13(17.6)$ & $91(16.8)$ \\
\hline LDH level data available, n (\%): & $250(55.1)$ & $325(58.0)$ & $68(54.0)$ & $643(56.4)$ \\
\hline - Elevated LDH level, n (\%) & $72(28.8)$ & $73(22.5)$ & $12(17.6)$ & $157(24.4)$ \\
\hline Stage available at first diagnosis, $\mathrm{n}(\%): \mathbb{I}$ & $340(74.9)$ & $440(78.6)$ & $106(84.1)$ & $886(77.7)$ \\
\hline - II or lower, $\mathrm{n}(\%)^{\dagger}$ & $121(35.6)$ & $153(34.8)$ & $37(34.9)$ & $311(35.1)$ \\
\hline - III, IIIA, IIIB, n (\%) ${ }^{\dagger}$ & $54(15.9)$ & $86(19.5)$ & $22(20.8)$ & $162(18.3)$ \\
\hline$-\mathrm{IIIC}, \mathrm{n}(\%)^{\dagger}$ & $39(11.5)$ & $37(8.4)$ & $10(9.4)$ & $86(9.7)$ \\
\hline$-I V, n(\%)^{\dagger}$ & $126(37.1)$ & $164(37.3)$ & $37(34.9)$ & $327(36.9)$ \\
\hline History of brain metastases, $\mathrm{n}(\%)$ : & $55(12.1)$ & $41(7.3)$ & $9(7.1)$ & $105(9.2)$ \\
\hline - Tested for NRAS & $86(18.9)$ & $110(19.6)$ & $10(7.9)$ & $206(18.1)$ \\
\hline - Positive NRAS status & $1(1.2)$ & $43(39.1)$ & $4(40.0)$ & $48(23.3)$ \\
\hline \multicolumn{5}{|c|}{$\begin{array}{l}\dagger \text { †atient percentages for race, region, ECOG PS, LDH level and stage at first diagnosis refer to those with available data. } \\
\text { ¥Weight data were missing for } 104(9 \%) \text { patients overall. } \\
\text { \$Included were } 20(8.0 \%), 21(6.5 \%) \text { and } 5(7.4 \%) \text { in the BRAF-mutant, BRAF wild-type and BRAF unknown cohorts, respectively, and } 46(7.2 \%) \text { overall, with LDH levels } \geq 2 \\
\text { times the upper limit of normal. } \\
\text { I Stage was a variable abstracted from the charts by trained abstractors; when missing, it was because of inadequate information available in the charts to determine stage } \\
\text { (including inadequate information in the doctor's handwritten notes, pathology reports, etc.). } \\
\text { CCI: Charlson Comorbidity Index; ECOG PS: Eastern Cooperative Oncology Group performance status; IQR: Interquartile Range; LDH: Lactate dehydrogenase; US CB: US Census } \\
\text { Bureau. }\end{array}$} \\
\hline
\end{tabular}

Time to next treatment line \& OS estimates

Table 4 summarizes the Kaplan-Meier estimates of time to next treatment line from first-line of therapy and overall by treatment category. The median time to next treatment line (including all treatment lines) for ipilimumab-based regimens was estimated at 4.9 months, anti-PD-1 monotherapy at 10.9 months, anti-PD-1 combination therapy at 8.2 months, BRAF inhibitor monotherapy at 4.7 months, BRAF inhibitor combination therapy at 6.8 months and other therapy at 4.5 months (Table 4).

From the time of initiation of first-line therapy, the Kaplan-Meier estimate of median OS for all patients was 18.8 months (95\% CI: 16.3-23.3) with 1-year, 2-year and 3-year estimated survival rates of 60.7, 45.3 and 35.0\%, respectively. The Kaplan-Meier estimate of median OS was 16.4 months (95\% CI: 14.0-21.4) in the BRAF-mutant cohort and 24.7 months (95\% CI: 18.4-not reached) in the BRAF wild-type cohort. 


\begin{tabular}{|c|c|c|c|c|}
\hline Characteristics & BRAF mutant $(n=454)$ & $B R A F$ wild-type $(n=560)$ & BRAF status unknown $(n=126)$ & Total $(n=1140)$ \\
\hline \multicolumn{5}{|l|}{ Number of treatment lines received, $\mathrm{n}(\%)$ : } \\
\hline$-1 \mathrm{~L}$ only & $259(57.0)$ & $379(67.7)$ & $96(76.2)$ & $734(64.4)$ \\
\hline$-1 L+2 L$ & $127(28.0)$ & $139(24.8)$ & $24(19.0)$ & $290(25.4)$ \\
\hline-3 or more lines & $68(15.0)$ & $42(7.5)$ & $6(4.8)$ & $116(10.2)$ \\
\hline \multicolumn{5}{|l|}{ Overview of treatment flow: ${ }^{\dagger}$} \\
\hline - Patients receiving $1 \mathrm{~L}$ therapy, $\mathrm{n}(\%)$ & $454(100)$ & $560(100)$ & $126(100)$ & $1140(100)$ \\
\hline - Completed 1L, n (\%) & $321(70.7)$ & $325(58.0)$ & 75 (59.5) & $721(63.2)$ \\
\hline - Advanced to $2 \mathrm{~L}, \mathrm{n}$ ( $\%$ of $1 \mathrm{~L}$ completers) & $195(60.7)$ & $181(55.7)$ & $30(40.0)$ & $406(56.3)$ \\
\hline - Completed $2 \mathrm{~L}, \mathrm{n}$ (\% of advancers) & $127(65.1)$ & $102(56.4)$ & $16(53.3)$ & $245(60.3)$ \\
\hline - Advanced to $3 \mathrm{~L}, \mathrm{n}$ (\% of $2 \mathrm{~L}$ completers) & $68(53.5)$ & $42(41.2)$ & $6(37.5)$ & $116(47.3)$ \\
\hline - Completed 3L, n (\% of advancers) & $46(67.6)$ & $24(57.1)$ & $5(83.3)$ & $75(64.7)$ \\
\hline - Advanced to $4 \mathrm{~L}, \mathrm{n}$ (\% of $3 \mathrm{~L}$ completers) & $17(37.0)$ & $13(54.2)$ & $2(40.0)$ & $32(42.7)$ \\
\hline Received targeted therapy, n (\%) & $319(70.3)$ & $7(1.3)$ & $7(5.6)$ & $333(29.2)$ \\
\hline Months of follow-up, ${ }^{\ddagger}$ median (IQR): & $10.4(5.4-16.8)$ & $10.4(4.3-16.8)$ & $7.3(2.6-13.2)$ & $9.9(4.3-16.4)$ \\
\hline - Range & $0.03-42.6$ & $0.3-37.9$ & $0.03-35.0$ & $0.03-42.6$ \\
\hline \multicolumn{5}{|c|}{$\begin{array}{l}\text { †Patients were considered to have completed a line of therapy if there was a next line of therapy or a } \geq 60 \text {-day gap to the last visit date or if the patient died. } \\
\text { ¥Months of follow-up from the index date (start of first-line therapy) until death or loss to follow-up. } \\
\text { 1L: First-line; 2L: Second-line; } 3 \mathrm{~L} \text { : Third-line; 4L: Fourth-line; IQR: Interquartile range. }\end{array}$} \\
\hline
\end{tabular}

Median (95\% CI) OS in months by first-line regimen type was 21.0 (15.7-28.7) for ipilimumab-based, 20.7 (19.7-not reached) for anti-PD-1 monotherapy, 17.4 (12.2-not reached) for anti-PD-1 combination therapy, 14.9 (8.8-31.0) for BRAF inhibitor monotherapy and 12.0 (9.1-15.7) for BRAF inhibitor combination therapy (Figure 3). The 1-year estimated survival rate by first-line regimen type was 62.0\% (95\% CI: 56.7-66.9\%) for ipilimumab-based, $67.5 \%$ (61.4-72.8\%) for anti-PD-1 monotherapy, 61.5\% (51.6-70.0\%) for anti-PD-1 combination therapy, $58.2 \%$ (45.2-69.2\%) for BRAF inhibitor monotherapy and 48.9\% (40.4-56.9\%) for BRAF inhibitor combination therapy.

\section{Discussion}

In this retrospective observational study, we observed that first-line treatment selection for AMel changed dramatically over the study period from 1 January 2014 through 30 June 2016. Specifically, ipilimumab-based and BRAF inhibitor monotherapy regimens were administered less frequently in the first-line setting after the third quarter of 2014, concurrent with FDA approval of anti-PD-1-based regimens beginning in the fourth quarter of 2014. The uptake of anti-PD-1 agents was observed in the $B R A F$-mutant cohort but was most dramatic for the $B R A F$ wild-type cohort, $91 \%$ of whom received an anti-PD-1 agent as monotherapy or combination therapy in the second quarter of 2016.

Observational data collected before the availability of immuno-oncology agents and BRAF inhibitor therapies are no longer relevant, as the treatment landscape and approved medicines are changing so quickly [43]. Several recent observational studies examine treatment patterns after the May 2011 approvals of ipilimumab and vemurafenib [28,44-47], but these observational data add only to our understanding of ipilimumab and do not reflect the incorporation of newer immuno-oncology agents.

In this descriptive, observational study, the median time to next treatment line was estimated overall at 10.9 months for anti-PD-1 monotherapy, 8.2 months for anti-PD-1 combination therapy and 6.8 months for BRAF inhibitor combination therapy. The median OS from initiation of first-line therapy for all patients was 18.8 months. The median OS for patients who received ipilimumab-based and anti-PD-1 monotherapy regimens was close to 2 years (21.0 and 20.7 months, respectively).

The median OS for first-line BRAF inhibitor combination therapy and first-line BRAF inhibitor monotherapy were 12.0 vs 14.9 months, respectively. This finding was unexpected. We reran the analyses at two institutions (Merck \& Co., Inc. and Flatiron Health), and the results remained consistent. We note that differences in OS between $B R A F$ cohorts and among regimens should be interpreted with caution because of the observational study design and because the OS results were not adjusted for baseline characteristics and may also be confounded by 

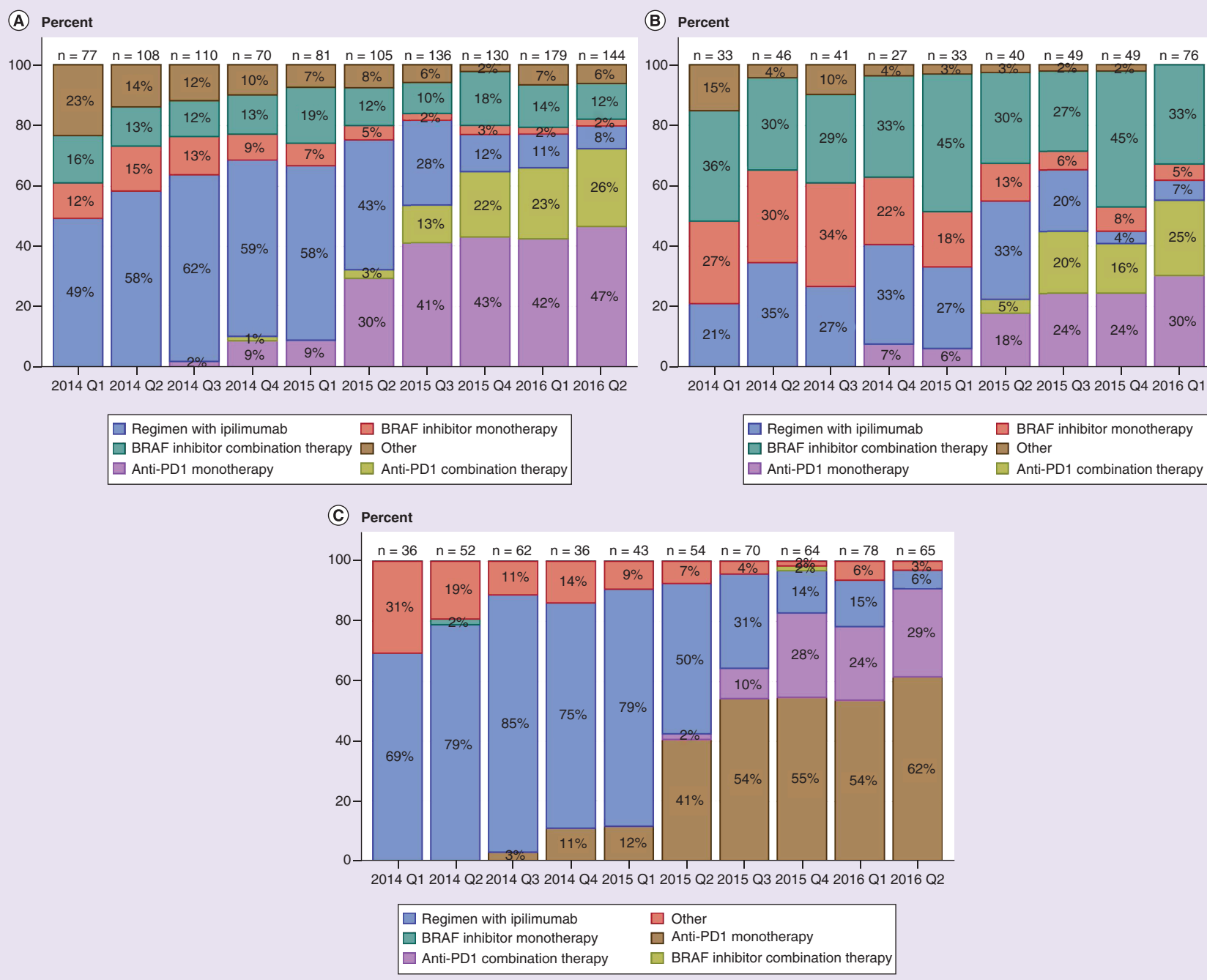

Figure 2. First-line therapy for patients with advanced melanoma from the first quarter of 2014 (2014Q1) through the second quarter of 2016 (2016Q2). (A) All patients with advanced melanoma $(n=1140)$; (B) patients with BRAF-mutant advanced melanoma $(n=454)$; (C) patients with $B R A F$ wild-type advanced melanoma $(n=560)$. Percentages are rounded and may not add up to $100 \%$.

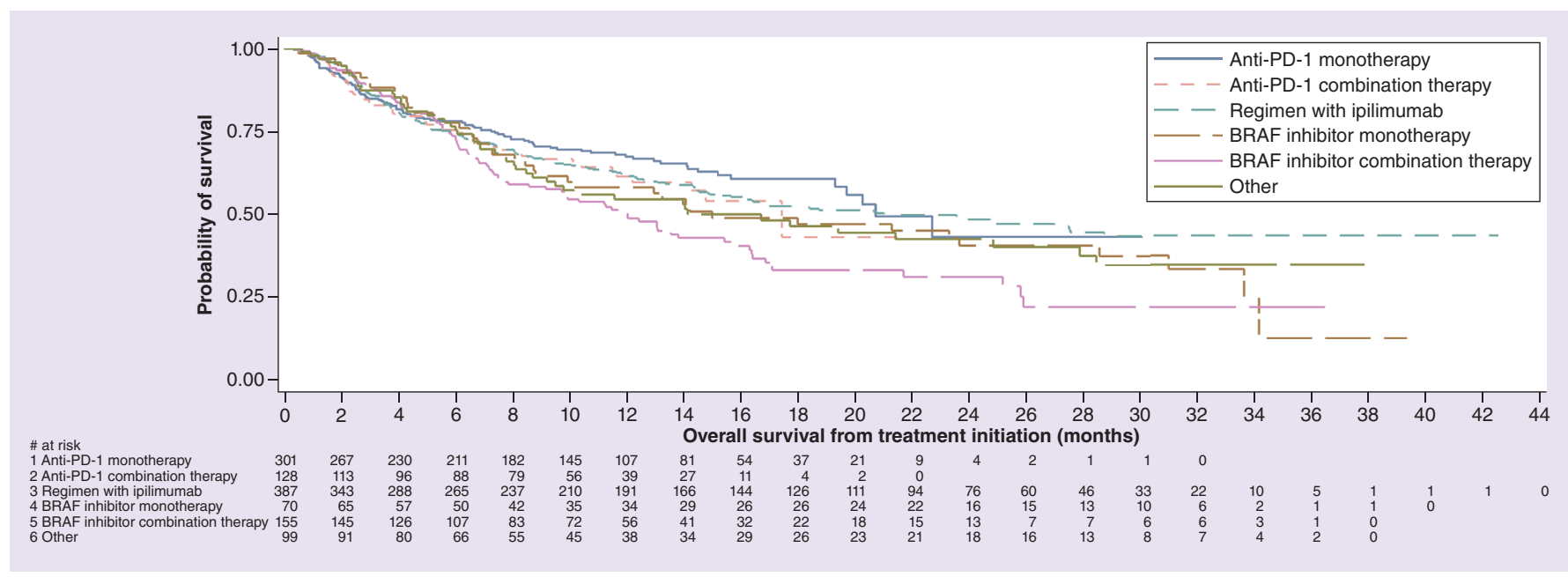

Figure 3. Kaplan-Meier plot for overall survival from initiation of first-line therapy start by regimen type. 


\begin{tabular}{|c|c|c|c|c|}
\hline Treatment category and regimen $^{\dagger}$ & BRAF mutant $(n=454)$ & BRAF wild-type $(\mathrm{n}=560)$ & BRAF status unknown $(\mathrm{n}=126)$ & Total $(n=1140)$ \\
\hline \multicolumn{5}{|l|}{ First-line therapy } \\
\hline Ipilimumab-based regimen & $85(18.7)$ & $254(45.4)$ & $48(38.1)$ & 387 (33.9) \\
\hline Ipilimumab & $69(15.2)$ & $247(44.1)$ & $48(38.1)$ & $364(31.9)$ \\
\hline Anti-PD-1 monotherapy & 76 (16.7) & 188 (33.6) & $37(29.4)$ & 301 (26.4) \\
\hline Pembrolizumab & $39(8.6)$ & $98(17.5)$ & $22(17.5)$ & $159(13.9)$ \\
\hline Nivolumab & $37(8.1)$ & $90(16.1)$ & $15(11.9)$ & $142(12.5)$ \\
\hline BRAF inhibitor regimen & 219 (48.2) & $2(0.4)$ & $4(3.2)$ & $225(19.7)$ \\
\hline Dabrafenib, trametinib & $135(29.7)$ & 0 & $2(1.6)$ & $137(12.0)$ \\
\hline Vemurafenib & $53(11.7)$ & 0 & $1(0.8)$ & $54(4.7)$ \\
\hline Dabrafenib & $13(2.9)$ & 0 & $0(0.0)$ & $13(1.1)$ \\
\hline Anti-PD-1 combination & $54(11.9)$ & $64(11.4)$ & $10(7.9)$ & $128(11.2)$ \\
\hline Ipilimumab, nivolumab & $37(8.1)$ & $60(10.7)$ & $9(7.1)$ & $106(9.3)$ \\
\hline Other & $20(4.4)$ & $52(9.3)$ & $27(21.4)$ & $99(8.7)$ \\
\hline Temozolomide & 0 & $17(3.0)$ & $1(0.8)$ & $18(1.6)$ \\
\hline Interferon & $7(1.5)$ & $5(0.9)$ & $2(1.6)$ & $14(1.2)$ \\
\hline Carboplatin, paclitaxel & $2(0.4)$ & $6(1.1)$ & $4(3.2)$ & $12(1.1)$ \\
\hline Second-line therapy & BRAF mutant $(\mathrm{n}=195)$ & BRAF wild-type $(\mathrm{n}=181)$ & $B R A F$ status unknown $(\mathrm{n}=30)$ & Total $(n=406)$ \\
\hline Anti-PD-1 monotherapy & $56(28.7)$ & $122(67.4)$ & $18(60.0)$ & $196(48.3)$ \\
\hline Pembrolizumab & $32(16.4)$ & $66(36.5)$ & $11(36.7)$ & 109 (26.8) \\
\hline Nivolumab & $24(12.3)$ & $56(30.9)$ & $7(23.3)$ & $87(21.4)$ \\
\hline BRAF/MEK inhibitor regimen & $76(39.0)$ & $2(1.1)$ & $1(3.3)$ & 79 (19.5) \\
\hline Dabrafenib, trametinib & $52(26.7)$ & $1(0.6)$ & $1(3.3)$ & $54(13.3)$ \\
\hline Vemurafenib & 7 (3.6) & 0 & 0 & $7(1.7)$ \\
\hline Vemurafenib, cobimetinib & $5(2.6)$ & 0 & 0 & $5(1.2)$ \\
\hline Ipilimumab-based regimen & $28(14.4)$ & $16(8.8)$ & $5(16.7)$ & $49(12.1)$ \\
\hline Ipilimumab & $23(11.8)$ & $15(8.3)$ & $5(16.7)$ & $43(10.6)$ \\
\hline Anti-PD-1 combination therapy & $27(13.8)$ & $18(9.9)$ & $2(6.7)$ & 47 (11.6) \\
\hline Ipilimumab, nivolumab & $15(7.7)$ & $12(6.6)$ & $1(3.3)$ & $28(6.9)$ \\
\hline Other & $8(4.1)$ & $23(12.7)$ & $4(13.3)$ & $35(8.6)$ \\
\hline Temozolomide & 0 & $8(4.4)$ & 0 & $8(2.0)$ \\
\hline \multicolumn{5}{|c|}{$\begin{array}{l}\text { †Regimens administered to } \geq 1 \% \text { of all patients are reported. } \\
\text { Regimen categories were defined as follows: anti-PD-1 monotherapy; anti-PD-1-combination: any regimen containing pembrolizumab or nivolumab; ipilimumab-based regimen: an } \\
\text { regimen containing ipilimumab but without an anti-PD-1 agent; BRAF/MEK inhibitor regimen: any regimen containing vemurafenib, trametinib, dabrafenib but without anti-PD- } \\
\text { agent or ipilimumab; other: regimens not included in the prior categories. }\end{array}$} \\
\hline
\end{tabular}

second-line therapies. For example, 39\% of patients who received ipilimumab-based regimens in first line received anti-PD-1 therapy in second line. In addition, follow-up is incomplete for some patients; many patients are receiving ongoing therapy and subsequent follow-up is planned. Further investigations are needed to explore the outcomes of various systemic therapies for AMel in the real-world setting.

Some data of clinical relevance that physicians may have considered in managing a patient's treatment were either limited or not available in the EHRs, including the number and location of sites of metastasis, disease progression, patient adherence to oral therapies, administration of regional therapies and information about adverse effects of therapy. Nonetheless, while some patient data were missing or incomplete, the cohort was well-characterized in many ways. Most patients (91\%) in our study had been tested for the BRAF mutation, as currently recommended for AMel [35], and we were able to describe longitudinal treatment patterns, including first- and subsequent lines of therapy, according to $B R A F$ mutation status. Almost half of patients tested in this study (45\%) had $B R A F$ mutant melanoma, within reported ranges for the US, Australia and Italy $[2,22,23]$, and on average, patients with $B R A F$-mutant tumors were younger than those with $B R A F$ wild-type melanoma, as reported in prior studies [22,48], suggesting our population was representative of a general AMel population. However, the patient population included in our study may differ from those included in randomized controlled trials in which patients are often 


\begin{tabular}{|c|c|c|c|}
\hline Treatment category & First-line therapy & Second-line and later therapy & All lines \\
\hline \multicolumn{4}{|l|}{ Anti-PD-1 monotherapy } \\
\hline Total patients, $\mathrm{N}$ & 301 & 255 & 556 \\
\hline Time (months) to next line, median $(95 \% \mathrm{Cl})$ : & $14.1(10.2-\mathrm{NA})$ & $7.4(5.1-11.7)$ & $10.9(8.4-14.3)$ \\
\hline - 1-year on the same line rate $(95 \% \mathrm{Cl})$ & $53.93 \%(47.68-59.76 \%)$ & $43.20 \%(36.53-49.67 \%)$ & $49.05 \%(44.49-53.44 \%)$ \\
\hline - 2-year on the same line rate $(95 \% \mathrm{Cl})$ & $40.51 \%(29.20-51.52 \%)$ & $33.37 \%(25.73-41.16 \%)$ & $37.42 \%(31.01-43.82 \%)$ \\
\hline \multicolumn{4}{|l|}{ Anti-PD-1 combination therapy } \\
\hline Total patients, $\mathrm{N}$ & 128 & 76 & 204 \\
\hline Time (months) to next line, median $(95 \% \mathrm{Cl})$ : & $11.3(6.4-\mathrm{NA})$ & $7.4(5.4-11.0)$ & $8.2(6.6-11.6)$ \\
\hline - 1-year on the same line rate $(95 \% \mathrm{Cl})$ & $45.47 \%(35.61-54.80 \%)$ & $29.66 \%(15.32-45.50 \%)$ & $41.13 \%(32.90-49.17 \%)$ \\
\hline \multicolumn{4}{|l|}{ Regimen with ipilimumab } \\
\hline Total patients, $\mathrm{N}$ & 387 & 60 & 447 \\
\hline Time (months) to next line, median $(95 \% \mathrm{Cl})$ : & $5.1(4.4-5.8)$ & $3.3(2.9-4.0)$ & $4.9(4.3-5.5)$ \\
\hline - 1-year on the same line rate $(95 \% \mathrm{Cl})$ & $27.73 \%(23.10-32.53 \%)$ & $13.95 \%(5.51-26.22 \%)$ & $26.05 \%(21.79-30.49 \%)$ \\
\hline - 2-year on the same line rate $(95 \% \mathrm{Cl})$ & $17.95 \%(13.74-22.64 \%)$ & $9.30 \%(2.28-22.49 \%)$ & $16.91 \%(13.04-21.23 \%)$ \\
\hline \multicolumn{4}{|l|}{ BRAF inhibitor monotherapy } \\
\hline Total patients, $\mathrm{N}$ & 70 & 23 & 93 \\
\hline Time (months) to next line, median $(95 \% \mathrm{Cl})$ : & $5.2(4.3-8.3)$ & $3.4(1.5-5.3)$ & $4.7(3.9-6.5)$ \\
\hline - 1-year on the same line rate $(95 \% \mathrm{Cl})$ & $27.32 \%(16.90-38.80 \%)$ & $20.50 \%(6.42-40.06 \%)$ & $25.25 \%(16.27-35.23 \%)$ \\
\hline - 2-year on the same line rate $(95 \% \mathrm{Cl})$ & $15.11 \%(7.10-25.91 \%)$ & NA & $12.96 \%(6.14-22.40 \%)$ \\
\hline \multicolumn{4}{|l|}{ BRAF inhibitor combination therapy } \\
\hline Total patients, $\mathrm{N}$ & 155 & 91 & 246 \\
\hline Time (months) to next line, median $(95 \% \mathrm{Cl})$ : & $6.8(5.8-9.1)$ & $6.6(5.5-9.0)$ & $6.8(6.0-7.9)$ \\
\hline - 1-year on the same line rate $(95 \% \mathrm{Cl})$ & $32.44 \%(24.69-40.42 \%)$ & $28.79 \%(18.87-39.47 \%)$ & $31.27 \%(25.07-37.65 \%)$ \\
\hline - 2-year on the same line rate $(95 \% \mathrm{Cl})$ & $10.98 \%(4.99-19.64 \%)$ & $10.52 \%(3.45-22.18 \%)$ & $10.57 \%(5.58-17.40 \%)$ \\
\hline \multicolumn{4}{|l|}{ Other } \\
\hline Total patients, $\mathrm{N}$ & 99 & 63 & 162 \\
\hline Time (months) to next line, median $(95 \% \mathrm{Cl})$ : & $6.0(4.3-9.7)$ & $3.2(2.2-4.2)$ & $4.5(3.8-6.0)$ \\
\hline - 1-year on the same line rate $(95 \% \mathrm{Cl})$ & $38.62 \%(28.60-48.53 \%)$ & $7.22 \%(1.91-17.46 \%)$ & $27.78 \%(20.43-35.59 \%)$ \\
\hline - 2-year on the same line rate $(95 \% \mathrm{CI})$ & $29.70 \%(19.77-40.28 \%)$ & NA & $19.44 \%(12.60-27.40 \%)$ \\
\hline
\end{tabular}

NA: Not available.

excluded if they have an ECOG PS of $\geq 2$ ( $17 \%$ of patients in our study) or active brain metastases ( $9 \%$ of patients in our study had a history of brain metastases) [31].

This study has several other limitations. Some of the data needed to characterize the patients' clinical and disease characteristics were incomplete, usually because the oncology clinic did not collect the information. For example, LDH level data at the index date were available for only $56 \%$ and ECOG PS data for only $48 \%$ of patients. We do not know why LDH was not assessed for all patients, but, as a result, we were unable to conduct multivariable analyses of $O S$ and time to next treatment line because these key variables were missing. Moreover, not all prescribing information for oral antineoplastic agents (e.g., trametinib, vemurafenib and dabrafenib) was available because of inconsistent recording in the medical record; thus, we made assumptions about prescription durations. For patients with multiple prescriptions of an oral drug, for example, trametinib, vemurafenib and dabrafenib, we calculated days' supply as the number of days between first and last prescriptions plus 30 days. For patients with only one prescription of an oral drug, we assumed 30 days' supply for those without such information. Moreover, while we were able to capture the variety of regimens prescribed in clinical practice, prescribing patterns may not be representative of those at different types of clinical practices, such as at academic medical centers, which are in the minority of practices participating with Flatiron. Detailed information regarding the management of brain metastases was not planned for this study. Future studies can potentially include this information for consideration, pending assessment of feasibility provided in real-world detailed documentation. 
Our findings suggest that NCCN guidelines for malignant melanoma are generally followed in community oncology practice [35]. Most patients with BRAF-mutant melanoma received targeted therapy with a BRAF inhibitor. Our findings also suggest that clinical practice is responsive to data presentation, inclusion in NCCN compendia, and label updates, as prescribing trends showed a drop in use of BRAF inhibitor monotherapy in favor of combination therapy and are now favoring the use of the anti-PD-1 agents, irrespective of $B R A F$ mutation status, with decreasing use of BRAF inhibitors and ipilimumab. There are more treatment choices for patients with $B R A F-$ mutant melanoma. Nonetheless, an unexpected finding was that BRAF inhibitor monotherapy was prescribed even after combination therapy was approved, perhaps because they are oral medications and associated in the US with higher out-of-pocket costs [49]. We can speculate that these changes are occurring because of perceived greater effectiveness of the anti-PD-1 agents, as evidenced in head-to-head clinical trials [20,31,32], coupled with the known frequent adverse effects associated with ipilimumab therapy [28,45,50,51].

More information is needed about the relationship between these changes and population-based outcomes such as contemporary survival estimates for AMel and patient experiences associated with these new therapies. Moreover, response patterns to immuno-oncology agents may differ from those to chemotherapy [52,53], underscoring the need for training to manage pseudoprogression and potential side effects from immunotherapy. Further studies are needed that include patients with all stages of melanoma and incorporate all treatment choices (e.g., surgery) into treatment patterns analysis to have a more complete understanding of the trajectory of clinical care over time.

\section{Conclusion}

The results of this study vividly illustrate the changing paradigms of therapy for AMel in US community oncology practices, with the increasing use of anti-PD-1 agents, regardless of BRAF mutation status. The time to next treatment line and OS findings provide a preliminary, descriptive assessment of real-world treatment outcomes but should be interpreted with caution because of the observational study design.

\section{Future perspective}

The treatment patterns for AMel in the real-world setting of US oncology clinical practices will likely continue to evolve with newer therapies available, and some of these therapies have now been approved for use in the adjuvant setting. In response to this trend, patient outcomes (e.g., OS) will likely continue to improve for patients with AMel.

\section{Acknowledgements}

The authors gratefully acknowledge the help of Y Zhong, J Feng and Q Xia (Merck \& Co., Inc., Kenilworth, NJ, USA) for providing statistical programming support. The current affiliation for X Cao is Novartis Pharmaceutical Co., East Hanover, NJ, USA.

\section{Authors' contributions}

Conception and design of the study: ED Whitman, FX Liu, AP Abernethy. Data analysis: X Cao. Interpretation of findings, critical review and revision of the manuscript: ED Whitman, FX Liu, X Cao, SJ Diede, A Haiderali, AP Abernethy. All authors read and approved the final manuscript.

\section{Financial \& competing interests disclosure}

This study was sponsored by Merck \& Co., Inc., Kenilworth, NJ, USA. The funder of the study participated in development of the study design and funded the analysis of the data. All authors, including those employed by Merck, participated in the data interpretation and writing of the manuscript. ED Whitman is a consultant and serves on advisory boards and/or speaker's bureaus for Merck, BMS, Genentech, Novartis, Amgen and Castle Biosciences. FX Liu, X Cao, SJ Diede and A Haiderali are employees and stockowners of Merck \& Co., Inc., Kenilworth, NJ, USA. AP Abernethy is Chief Medical Officer, Chief Scientific Officer and SVP, Oncology at Flatiron Health, a member of the Roche Group and has stock ownership in Roche. AP Abernethy also declares the following: Board of Directors and stock ownership of Athenahealth and CareDx, owner of Orange Leaf Associates, Senior Advisor of Highlander Partners, advisor of SignalPathResearch, RobinCare and KelaHealth, Inc., special advisor of The One Health Company, honoraria from Roche/Genentech (<US\$10K per year) and patent pending for a technology that facilitates the extraction of unstructured information from medical records. The authors have no other relevant affiliations or financial involvement with any organization or entity with a financial interest in or financial conflict with the subject matter or materials discussed in the manuscript apart from those disclosed. 
Medical writing and editorial assistance was provided by EV Hillyer, DVM. This assistance was funded by Merck Sharp \& Dohme Corp., a subsidiary of Merck \& Co., Inc., Kenilworth, NJ, USA.

\section{Ethical conduct of research}

The de-identified dataset supporting the conclusions of this article was provided by Flatiron Health. Institutional review board (IRB) approval of the study protocol was obtained through Flatiron procedure and approved by Copernicus Group IRB before study conduct and included a waiver of informed consent. Provisions were in place to prevent re-identification of de-identified data in order to protect patients' confidentiality.

\section{Open access}

This work is licensed under the Attribution-NonCommercial-NoDerivatives 4.0 Unported License. To view a copy of this license, visit http://creativecommons.org/licenses/by-nc-nd/4.0/

\section{Summary points}

- The last 8 years have seen incredible growth in the number of systemic therapies available for advanced melanoma (AMel). However, limited information exists on how these trends and associated patient outcomes are manifesting in a real-world setting.

- This study aimed to understand the evolution of treatment patterns, time to next treatment line and associated overall survival for patients with AMel in US real-world oncology clinical practices.

- In total, 1140 patients with AMel from a longitudinal and geographically diverse electronic health record database were included for this analysis.

- The most common first-line regimens were ipilimumab-based therapies (34\%), followed by anti-PD-1 monotherapy (26\%) and regimens including a BRAF inhibitor (20\%).

- Since the third quarter of 2014, when the US FDA first approved an anti-PD-1 agent for systemic therapy of AMel, the treatment regimens have changed rapidly, with decreased first-line prescribing of ipilimumab-based and BRAF inhibitor regimens, and by the second quarter of 2016, 55 and $91 \%$ of BRAF-mutant and BRAF wild-type cohorts, respectively, received a first-line anti-PD-1 regimen.

- The median time to next treatment across all treatment lines was 4.9 months for ipilimumab-based regimens, 10.9 and 8.2 months for anti-PD-1 monotherapy and combination therapy, respectively, and 4.7 and 6.8 months for BRAF inhibitor monotherapy and BRAF/MEK inhibitor combination therapy, respectively.

- The 1-year estimated survival rate by first-line regimen type was $62.0 \%$ for ipilimumab-based, $67.5 \%$ for anti-PD-1 monotherapy, $61.5 \%$ for anti-PD-1 combination therapy, 58.2\% for BRAF inhibitor monotherapy and $48.9 \%$ for BRAF/MEK inhibitor combination therapy. The median OS from initiation of first-line therapy for all patients was 18.8 months (95\% Cl: 16.3-23.3).

- The study results illustrate the rapidly changing paradigms of systemic therapy for AMel in US clinical oncology practices, with increasing use of anti-PD-1 agents, regardless of BRAF mutation status.

- The findings on time to next treatment line and overall survival provide a preliminary, descriptive assessment of real-world treatment outcomes for patients with AMel, and further research should be conducted to confirm these results.

\section{References}

Papers of special note have been highlighted as: $\bullet$ of interest

1. AIM at Melanoma Foundation. Melanoma stats, facts, and figures. www.aimatmelanoma.org/about-melanoma/melanoma-stats-facts-and-figures/

2. Eggermont AM, Spatz A, Robert C. Cutaneous melanoma. Lancet 383(9919), 816-827 (2014).

- Comprehensive review article on melanoma epidemiology, diagnosis and therapy.

3. Schoffer O, Schulein S, Arand G et al. Tumour stage distribution and survival of malignant melanoma in Germany 2002-2011. BMC Cancer 16(1), 936 (2016).

4. SEER cancer statistics factsheets: melanoma of the skin. http://seer.cancer.gov/statfacts $/ \mathrm{html} / \mathrm{melan} . \mathrm{html}$

5. Noone AM, Howlader N, Krapcho M et al. (Eds). SEER cancer statistics review, 1975-2015, based on November 2017 SEER data submission (2018). National Cancer Institute, MD, USA. https://seer.cancer.gov/csr/1975_2015/

6. Ives NJ, Stowe RL, Lorigan P, Wheatley K. Chemotherapy compared with biochemotherapy for the treatment of metastatic melanoma: a meta-analysis of 18 trials involving 2,621 patients. J. Clin. Oncol. 25(34), 5426-5434 (2007).

7. Serrone L, Zeuli M, Sega FM, Cognetti F. Dacarbazine-based chemotherapy for metastatic melanoma: thirty-year experience overview. J. Exp. Clin. Cancer Res. 19(1), 21-34 (2000). 
8. Lorigan P, Marples M, Harries $\mathrm{M}$ et al. Treatment patterns, outcomes, and resource utilization of patients with metastatic melanoma in the UK: the MELODY study. Br. J. Dermatol. 170(1), 87-95 (2014).

9. Lebbe C, Lorigan P, Ascierto P et al. Treatment patterns and outcomes among patients diagnosed with unresectable stage III or IV melanoma in Europe: a retrospective, longitudinal survey (MELODY study). Eur. J. Cancer 48(17), 3205-3214 (2012).

10. Salama AK, Rosa N, Scheri RP et al. The effect of metastatic site and decade of diagnosis on the individual burden of metastatic melanoma: contemporary estimates of average years of life lost. Cancer Invest. 30(9), 637-641 (2012).

11. Atkins MB, Lotze MT, Dutcher JP et al. High-dose recombinant interleukin 2 therapy for patients with metastatic melanoma: analysis of 270 patients treated between 1985 and 1993. J. Clin. Oncol. 17(7), 2105-2116 (1999).

12. Kaufman HL, Kirkwood JM, Hodi FS et al. The Society for Immunotherapy of Cancer consensus statement on tumour immunotherapy for the treatment of cutaneous melanoma. Nat. Rev. Clin. Oncol. 10(10), 588-598 (2013).

13. McArthur GA, Ribas A. Targeting oncogenic drivers and the immune system in melanoma. J. Clin. Oncol. 31(4), 499-506 (2013).

14. Hodi FS, O'Day SJ, McDermott DF et al. Improved survival with ipilimumab in patients with metastatic melanoma. $N$. Engl. J. Med. 363(8), 711-723 (2010).

15. Robert C, Thomas L, Bondarenko I et al. Ipilimumab plus dacarbazine for previously untreated metastatic melanoma. $N$. Engl. J. Med. 364(26), 2517-2526 (2011).

16. Chapman $\mathrm{PB}$, Hauschild A, Robert $\mathrm{C}$ et al. Improved survival with vemurafenib in melanoma with BRAF V600E mutation. $N$. Engl. J. Med. 364(26), 2507-2516 (2011).

17. McArthur GA, Chapman PB, Robert $\mathrm{C}$ et al. Safety and efficacy of vemurafenib in BRAF(V600E) and BRAF(V600K) mutation-positive melanoma (BRIM-3): extended follow-up of a Phase III, randomised, open-label study. Lancet Oncol. 15(3), 323-332 (2014).

18. Hauschild A, Grob JJ, Demidov LV et al. Dabrafenib in BRAF-mutated metastatic melanoma: a multicentre, open-label, Phase III randomised controlled trial. Lancet 380(9839), 358-365 (2012).

19. Robert C, Karaszewska B, Schachter J et al. Improved overall survival in melanoma with combined dabrafenib and trametinib. N. Engl. J. Med. 372(1), 30-39 (2015).

20. Robert C, Schachter J, Long GV et al. Pembrolizumab versus ipilimumab in advanced melanoma. N. Engl. J. Med. 372(26), 2521-2532 (2015).

21. Robert C, Long GV, Brady B et al. Nivolumab in previously untreated melanoma without BRAF mutation. N. Engl. J. Med. 372(4), 320-330 (2015).

22. Menzies AM, Haydu LE, Visintin L et al. Distinguishing clinicopathologic features of patients with V600E and V600K BRAF-mutant metastatic melanoma. Clin. Cancer Res. 18(12), 3242-3249 (2012).

23. Colombino $\mathrm{M}$, Lissia $\mathrm{A}$, Capone $\mathrm{M}$ et al. Heterogeneous distribution of BRAF/NRAS mutations among Italian patients with advanced melanoma. J. Transl. Med. 11, 202 (2013).

24. Larkin J, Ascierto PA, Dreno B et al. Combined vemurafenib and cobimetinib in BRAF-mutated melanoma. N. Engl. J. Med. 371(20), 1867-1876 (2014).

- Randomized Phase III clinical trial investigating the efficacy and safety of combining BRAF and MEK inhibition for treating BRAF V600-mutated metastatic melanoma.

25. Flaherty KT, Infante JR, Daud A et al. Combined BRAF and MEK inhibition in melanoma with BRAF V600 mutations. N. Engl. J. Med. 367(18), 1694-1703 (2012).

- Early, open-label study examining combined BRAF and MEK inhibition for treating BRAF V600-mutated metastatic melanoma.

26. Long GV, Stroyakovskiy D, Gogas $\mathrm{H}$ et al. Dabrafenib and trametinib versus dabrafenib and placebo for Val600 BRAF-mutant melanoma: a multicentre, double-blind, Phase III randomised controlled trial. Lancet 386(9992), 444-451 (2015).

27. Ribas A, Puzanov I, Dummer R et al. Pembrolizumab versus investigator-choice chemotherapy for ipilimumab-refractory melanoma (KEYNOTE-002): a randomised, controlled, Phase II trial. Lancet Oncol. 16(8), 908-918 (2015).

28. Ahmad SS, Qian W, Ellis $S$ et al. Ipilimumab in the real world: the UK expanded access programme experience in previously treated advanced melanoma patients. Melanoma Res. 25(5), 432-442 (2015).

29. McDermott D, Haanen J, Chen TT, Lorigan P, O’Day S. Efficacy and safety of ipilimumab in metastatic melanoma patients surviving more than 2 years following treatment in a Phase III trial (MDX010-20). Ann. Oncol. 24(10), 2694-2698 (2013).

30. Hamid O, Puzanov I, Dummer R et al. Final analysis of a randomised trial comparing pembrolizumab versus investigator-choice chemotherapy for ipilimumab-refractory advanced melanoma. Eur. J. Cancer 86, 37-45 (2017).

31. Schachter J, Ribas A, Long GV et al. Pembrolizumab versus ipilimumab for advanced melanoma: final overall survival results of a multicentre, randomised, open-label Phase III study (KEYNOTE-006). Lancet 390(10105), 1853-1862 (2017).

- The final protocol-specified survival analysis for KEYNOTE-006 study, with median follow-up of 22.9 months $(\mathrm{n}=811)$.

32. Larkin J, Minor D, D'Angelo S et al. Overall survival in patients with advanced melanoma who received nivolumab versus investigator's choice chemotherapy in CheckMate 037: a randomized, controlled, open-label Phase III trial. J. Clin. Oncol. 36(4), 383-390 (2018).

- Overall survival (OS) endpoint results of CheckMate-037 study, with follow-up of approximately 2 years $(\mathrm{n}=405)$. 
33. Wolchok JD, Chiarion-Sileni V, Gonzalez R et al. Overall survival with combined nivolumab and ipilimumab in advanced melanoma. N. Engl. J. Med. 377(14), 1345-1356 (2017).

- $\quad$ OS outcomes $(n=945)$ of CheckMate-067 study, with minimum follow-up of 3 years for living patients.

34. Sznol M, Ferrucci PF, Hogg D et al. Pooled analysis safety profile of nivolumab and ipilimumab combination therapy in patients with advanced melanoma. J. Clin. Oncol. 35(34), 3815-3822 (2017).

35. NCCN clinical practice guidelines in oncology: melanoma. www.nccn.org/professionals/physician_gls/f_guidelines.asp

36. Mitchell AP, Harrison MR, Walker MS, George DJ, Abernethy AP, Hirsch BR. Clinical trial participants with metastatic renal cell carcinoma differ from patients treated in real-world practice. J. Oncol. Pract. 11(6), 491-497 (2015).

37. Miller RS, Wong JL. Using oncology real-world evidence for quality improvement and discovery: the case for ASCO's CancerLinQ. Future Oncol. 14(1), 5-8 (2018).

38. Flatiron Health: flatiron database.www.flatiron.com/real-world-evidence

39. Centers for Disease Control and Prevention. International Classification of Diseases, Ninth Revision, Clinical Modification (ICD-9-CM). www.cdc.gov/nchs/icd/icd $9 \mathrm{~cm} . h t m$

40. Quan H, Sundararajan V, Halfon P et al. Coding algorithms for defining comorbidities in ICD-9-CM and ICD-10 administrative data Med. Care. 43(11), 1130-1139 (2005).

41. Social Security Death Master File. www.ssdmf.com/

42. Quinn J, Kramer N, McDermott D. Validation of the Social Security Death Index (SSDI): an important readily-available outcomes database for researchers. West. J. Emerg. Med. 9(1), 6-8 (2008).

43. Harries M, Malvehy J, Lebbe C et al. Treatment patterns of advanced malignant melanoma (stage III-IV) - a review of current standards in Europe. Eur. J. Cancer 60, 179-189 (2016).

44. Toy EL, Vekeman F, Lewis MC, Oglesby AK, Duh MS. Costs, resource utilization, and treatment patterns for patients with metastatic melanoma in a commercially insured setting. Curr. Med. Res. Opin. 31(8), 1561-1572 (2015).

45. Ascierto PA, Simeone E, Sileni VC et al. Clinical experience with ipilimumab $3 \mathrm{mg} / \mathrm{kg}$ : real-world efficacy and safety data from an expanded access programme cohort. J. Transl. Med. 12, 116 (2014).

46. Mohr P, Ascierto P, Arance A et al. Real-world treatment patterns and outcomes among metastatic cutaneous melanoma patients treated with ipilimumab. J. Eur. Acad. Dermatol. Venereol. 32(6), 962-671 (2018).

47. Forschner A, Eichner F, Amaral T, Keim U, Garbe C, Eigentler TK. Improvement of overall survival in stage IV melanoma patients during 2011-2014: analysis of real-world data in 441 patients of the German Central Malignant Melanoma Registry (CMMR). J. Cancer Res. Clin. Oncol. 143(3), 533-540 (2017).

48. Bauer J, Buttner P, Murali R et al. BRAF mutations in cutaneous melanoma are independently associated with age, anatomic site of the primary tumor, and the degree of solar elastosis at the primary tumor site. Pigment Cell Melanoma Res. 24(2), 345-351 (2011).

49. Doshi JA, Li P, Huo H, Pettit AR, Armstrong KA. Association of patient out-of-pocket costs with prescription abandonment and delay in fills of novel oral anticancer agents. J. Clin. Oncol. 36(5), 476-482 (2018).

50. Delyon J, Mateus C, Lefeuvre $\mathrm{D}$ et al. Experience in daily practice with ipilimumab for the treatment of patients with metastatic melanoma: an early increase in lymphocyte and eosinophil counts is associated with improved survival. Ann. Oncol. 24(6), 1697-1703 (2013).

51. Alexander M, Mellor JD, McArthur G, Kee D. Ipilimumab in pretreated patients with unresectable or metastatic cutaneous, uveal and mucosal melanoma. Med. J. Aust. 201(1), 49-53 (2014).

52. Chiou VL, Burotto M. Pseudoprogression and immune-related response in solid tumors. J. Clin. Oncol. 33(31), 3541-3543 (2015).

53. Hodi FS, Hwu WJ, Kefford R et al. Evaluation of immune-related response criteria and RECIST v1.1 in patients with advanced melanoma treated with pembrolizumab. J. Clin. Oncol. 34(13), 1510-1517 (2016).

- Comparison of the relationship between OS and best overall response as measured per immune-related response criteria versus the Response Evaluation Criteria in Solid Tumors, version 1.1 (RECIST v1.1). 
